\title{
Comparison Analysis of Rapid Assessment Models for Earthquake Disaster Life Loss in Gansu Province for Earthquake Emergency Response
}

\author{
Wen Li, Wenkai Chen, Zhonghong Zhou, Yanping Sun, Jin Chen \\ Lanzhou Institute of Seismology, CEA, Lanzhou 730000, China
}

\section{面向地震应急的甘肃省地震灾害生命损失快速 评估模型对比研究 \\ 李雯, 陈文凯, 周中红, 孙艳萍, 陈晋 \\ 中国地震局兰州地震研究所, 兰州 730000 , 中国}

\begin{abstract}
In this paper, a typical life loss model for earthquake disasters is used to verify and calculate the historical earthquake cases in Gansu Province since 1966. The accuracy and applicability of various models in Gansu Province are studied through actual earthquake cases. The study results shows that: (1) when the earthquake magnitude is lower than 5.5 or between 5.5 and 6 , The live loss assessment model based on population density proposed by Badal, the assessment model of life loss based on epicenter intensity proposed by Liu Jinlong, and the national emergency assessment model for earthquake disasters are show minimum-error and have the best comprehensive effect; When the earthquake magnitude is higher than 6 , needs to analyze the characteristics of the natural geography, the social economy and other aspects of the assessment area to get the comprehensive evaluation results to support the emergency decision. (2)The assessment results of the life loss model of seismic disaster in the space are
\end{abstract}

obvious regionally: The accuracy of the earthquake model evaluation in the Hedong area of Gansu is not as accurate as that in the Hexi region. In the research of life loss assessment model for earthquake disasters, it is particularly important to build a typical assessment model for regional seismic loss characteristics. In addition, the prospects on the problems in the current life vulnerability models in earthquake disasters and the future development are presented.

Keywords:Earthquake disaster; Life losses; Rapid assessment; Applicability evaluation

\section{摘要}

本文采用典型地震灾害生命损失评估 模型, 以 1966 年以来甘肃省历史震例验证 计算, 通过实际震例研究各类模型在甘肃 省的评估精度和适用性, 结果表明: (1)震 级 $M_{s}<5.5 、 5.5 \leqslant M_{s} \leqslant 6.0$ 级地震 Badal 提 出的以人口密度划分的生命损失评估模型、 刘金龙提出的基于震中烈度的生命损失评 估模型以及国家地震灾情应急评估模型相 对较为准确, 基本都在合理范围内; 震级 
$M s>6.0$ 级地震需对评估区域的自然地理、 社会经济等方面存在的特征进行分析得到 综合评估结果来支撑应急决策。(2)在空间 上地震灾害生命损失评估模型存分区现象, 评估结果存在明显的区域性: 甘肃河东地 区发生地震模型评估结果准确性不如河西 地区。在地震灾害生命损失评估模型研究 中, 构建针对区域性地震生命损失特征的 典型评估模型尤为重要。此外本文对目前 地震灾害生命易损性模型存在的问题及解 决途径进行了讨论。

关键词: 地震灾害; 生命损失; 快速评估; 适用性评价

\section{1. 引言}

我国是世界上地震灾害最严重的国家 之一, 而甘肃省处于南北地震带北段, 是 我国多震省份之一。据记载 8.0 级及以上 地震甘肃省发生过 4 次, 造成了大量的人 员伤亡, 其中 1920 年海原发生 8.5 级地震, 导致 $273465 \pm 9700$ 人死亡 ${ }^{[1]} ; 1927$ 年古浪 发生 8.0 级地震, 导致 41471 人死亡。近 年来甘肃省及其周边地区地震活动增强, 如四川汶川 8.0 级地震、四川九寨沟 7.0 级地震、甘肃岷县漳县 6.6 级地震、青海 门源 6.4 级地震等 ${ }^{[2]}$ 。由于地震特有的瞬时 突发性, 大地震往往会造成严重的生命损 失。

根据中国《自然灾害救助条例》, 在自 然灾害的救助工作中, “以人为本” 是首 要遵循的原则 (中华人民共和国国务院, 2010 )。此外在实际地震应急响应中, 响应 级别与救灾物资呈正比; 如果应急响应级 别过高, 会导致救援物资的大量浪费, 如 果偏低则不利于灾后救助。“人员伤亡” 作为地震应急响应级别启动的一个重要因 素, 直接决定着政府地震应急响应启动级 别 (《国家地震应急预案》, 2012)。地震灾 害生命损失快速评估是实际地震应急亟需 解决的一个科学问题, 开展面向地震应急 的生命损失快速评估模型研究可以第一时 间为各级政府应急指挥决策提供技术支持, 地震灾害生命损失快速评估研究具有非常 重要的科学意义和现实意义。本文采用典
型地震灾害生命损失评估模型, 以 1966 年 以来甘肃省历史震例验证计算, 通过实际 震例研究各类模型在甘肃省的评估精度、 适用性及存在的问题, 探讨解决问题的途 径。

\section{2. 研究进展}

面向地震应急的地震灾害生命损失评 估研究目前主要有 2 大类评估模型 ${ }^{[3]}$ : 一是 通过建筑易损性分析得到的地震人员死亡 率模型。这里一般包括基于历史资料和专 家经验的震害矩阵法和基于性能的易损性 分析方法, 如尹之潜 ${ }^{[4]}$ 及其研究小组提出了 建筑物及设施的地震易损性分类方法、各 类房屋结构的易损性分析方法、地震灾害 损失预测方法; 何玉林 ${ }^{[5]}$ 通过对四川省境内 10 例地震震例的经验统计得到四川省不同 地区的房屋建筑易损性矩阵; FEMA 委托美 国国家建筑科学研究院 (National Institute of Building Sciences, NIBS) 经过几年的理论研究和论证, 于 1997 年推 出了一套标准化的地震损失预测方法 HAZUS97 ${ }^{[6]}$ 。前者对承灾体载体的数据详细 程度要求程度不高, 但是对建筑类别的划 分比较粗, 受当地地震历史条件的限制, 震害矩阵的准确度和可信度会受到影响; 后者充分考虑了结构类型和震后损伤状态 对伤亡的影响, 对地震人员伤亡进行了系 统的计算, 缺点是需要对地区的建筑进行 详细分类和细致调查, 并建立详细的建筑 数据库, 进行大量的数值计算和统计分析 ${ }^{[7]}$ 。 二是不考虑建筑破坏情况, 通过回归分析 历史震害数据得到的基于地震参数（震级、 烈度等) 的人员死亡数或死亡率经验公式。 第一类以人口密度为划分标准, 如 Samardjieva 等 ${ }^{[8]}$ 人对全球 1990 年以来的 地震案例进行研究, 以人口密度为划分标 准 $\left(25\right.$ 人 $/ \mathrm{km}^{2}$ 以下; $25 \sim 50$ 人 $/ \mathrm{km}^{2} ; 50 \sim$ 100 人 $/ \mathrm{km}^{2} ; 100 \sim 200$ 人 $/ \mathrm{km}^{2} ; 200$ 人 $/ \mathrm{km}^{2}$ 以上), 得到了全球尺度下 $1900^{\sim} 1950$ 年和 $1950^{\sim} 1999$ 年的地震死亡数与震级经验公 式; 在此基础上 Bada 1 等 $^{[9]}$ 构建了利用震级、 人口密度等指标评估人员伤亡的模型, 并 用来评估西班牙 8 个城市假设遭遇 6 级和 6. 5 级地震时的人员伤亡数量; 第二类以 
时间为划分标准, 陈棋福 ${ }^{[10]}$ 对国内 $1980--$ 2000 年的地震案例进行了研究, 将地震发 生的时间分为白天夜晚两个时段, 得到了 以人口密度为划分标准的人员死亡数与烈 度有关的经验公式; 第三类以人均 GDP 为 划分标准, 陈颙等人对 1989 年到 2004 年 的 207 例地震案例进行了研究, 以 2000 年 不变价格表示的人均 GDP 2700 元为分类阈 值, 得到生命损失率与地震烈度的关系; 刘吉夫等根据之前提出的宏观易损性研究 思路, 按照地震实际烈度来分配人口和 GDP, 建立了更加接近地震实情的地震生命易损 性模型 ${ }^{[11][12]}$ 。基于地震参数的经验公式基 于统计规律, 对历史数据的依赖性比较强, 选取的历史数据集合不同对建立的计算方 法会产生很大影响, 建模时主要影响因素 的选取是造成各种模型评估结果差异的主 因, 但简单的参数获取比较适用于震后人 员伤亡快速评估的需要。因此, 在应对震 后人员快速评估的需要时, 经验公式是一 个比较理想的模型。

\section{3. 资料收集与处理}

考虑到地震灾害数据的可靠性和完整性, 本文选取了 1966 年以来凡是正式开展了地 震现场震害调查和损失评估的甘肃省地震 灾害损失评估报告, 包括从中国大陆地震 灾害损失评估汇编（1990-1995）（19962000)、(2001-2005)、(2006-2010)、 (2011-2015) 中搜集甘肃震例损失数据

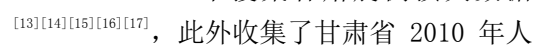
口普查分乡、镇、街道资料、历年甘肃统 计年鉴等数据。通过资料分析, 选取了最 高烈度在 6 度及以上 16 例成灾地震的震例 用于地震灾害生命损失评估模型的验证并 提取相关地震参数, 包括发震日期、发震 时刻、经纬度坐标、震级和死亡人数。

对篮选出的 16 个地震的烈度图逐一扫 描, 利用美国 ESRI 公司开发的 ArcGIS 软 件对烈度图进行配准和数字化, 得到一次 地震各个烈度区的面积及分布范围。在以 往的部分研究中人口数据都是分配在地震 宏观震中烈度 (最高烈度), 这和实际地震 情况会有较大差别, 同时实际生命损失分 布与人口在烈度圈的分布密切相关。本文
以实际烈度区作为分配单元, 根据地震发 生时各烈度区所涉及的乡镇人口, 按各烈 度区中乡镇面积占比换算得到分烈度区人 口数量。

\section{4. 评估模型选择}

在收集整理 2000 年以来公开发表的地 震生命损失评估模型时发现, 不同模型来 源不同、所采用的模型表达方式不同、适 用的空间范围和时间段也不同。考虑到基 于房屋易损性的人员伤亡评估方法需要较 为精确的建筑物分类及地震房屋倒塌率, 并遵循适用于震后死亡人口快速评估、所 用参数易获取、评估结果明晰等原则, 确 定了基于地震参数的人员死亡经验模型大 类中 4 小类地震灾害生命易损性模型（模 型名称由人名代替) 作为研究的评估模型。

1、以人口密度为划分标准的生命易损 性模型

选取 Badal 等 $^{[9]}$ 构建了利用震级、人口 密度等指标评估人员伤亡的模型, 模型较 为简单易推广, 其公式为:

$$
\log N_{k}(D)=a(D)+b(D) M
$$

式中, $M$ 表示震级; $D$ 表示人口密度; $N_{k}$ 分别为预测的死亡人数。将人口密度划 分为 25 人 $/ \mathrm{km}^{2}$ 以下; $25 \sim 50$ 人/ $\mathrm{km}^{2}$; $50 \sim 100$ 人 $/ \mathrm{km}^{2} ; 100 \sim 200$ 人/ $\mathrm{km}^{2} ; 200$ 人/ $\mathrm{km}^{2}$ 以上, 不同人口密度等级, $\mathrm{a}, \mathrm{b}$ 参 数取值不同。

2、以宏观经济指标为划分标准的生命 易损性模型

刘吉夫等 ${ }^{[12]}$ 人根据之前提出的宏观易 损性研究思路, 以 2000 年不变价格计算的 人均 GDP 值 2700 元为分类阈值, 将所有统 计数据分为 2 组, 进行回归统计:

$$
R_{M D F}=C A I^{B}
$$

式中, $R_{M D F}$ 代表生命损失率; I 为地 震烈度; $A, B$ 为系数; $C$ 为修正系数。该 模型中估算的是生命损失率, 在实际应用 中需要获取受灾人口, 最终得到地震死亡 人数。

3、基于震中烈度的生命易损性模型

刘金龙等 ${ }^{[18]}$ 人以我国 1990-2006 年间 造成人员伤亡的破坏性地震为数据样本, 结合国内几次大地震的震害资料, 找出了 
影响人员伤亡的主要因素, 经过高斯函数 拟合与回归分析, 得到了一个以震中烈度 为主要参数, 以震级和人口密度作为辅助 参数进行修正的人员伤亡预测模型, 最终 得到的伤亡模型如下:

$$
\mathrm{D}=e^{12.2 \alpha_{d e n} \alpha_{m} \cdot e^{-(\ln (\ln t)-2.445)^{2} / 0.3^{2}}}
$$

式中, D 代表死亡数量; $\ln t$ 表示震中 烈度; $\alpha_{m}$ 为震级修正系数; $\alpha_{d e n}$ 为人口密 度修正系数。

4. 国家标准 GB/T 30352-2013 地震
次震中烈度 6 度以上的地震人员死亡情况 进行评估, 将评估结果与地震实际造成人 员死亡数量比较, 为方便统计, 本文将 16 例地震作为样本并进行编号, 评估结果如 表 1 所示。

从评估结果来看, 4 种模型相对误差值 浮动变化明显, 预测死亡人数波动较大, 有些案例评估结果与实际结果较为一致, 有些案例的多数评估结果与实际结果基本 不属于一个数量级, 差异明显。为进一步 验证 4 种模型整体评估结果的准确性, 本

表 1 四类模型评估的结果与实际死亡人数对比

\begin{tabular}{|c|c|c|c|c|c|c|c|c|}
\hline $\mathrm{ID}$ & Date & 震级 & 极震区地名 & 死亡/人 & 刘吉夫 & Badal & $\begin{array}{c}\text { GB/T30352- } \\
2013\end{array}$ & 刘金龙 \\
\hline 1 & 19840106 & 5.5 & 天祝 & 0 & 1 & 2 & 0 & 1 \\
\hline 2 & 19870108 & 5.9 & 迭部 & 0 & 34 & 5 & 2 & 2 \\
\hline 3 & 19871025 & 5 & 礼县 & 0 & 14 & 4 & 0 & 1 \\
\hline 4 & 19881122 & 5.7 & 肃南 & 0 & 0 & 2 & 0 & 1 \\
\hline 5 & 19901020 & 6.2 & 天祝 & 1 & 26 & 9 & 2 & 9 \\
\hline 6 & 19920112 & 5.4 & 肃南 & 0 & 2 & 2 & 0 & 1 \\
\hline 7 & 19950722 & 5.8 & 永登 & 12 & 117 & 11 & 14 & 12 \\
\hline 8 & 19960601 & 5.4 & 天祝-古浪 & 0 & 30 & 6 & 1 & 2 \\
\hline 9 & 19990415 & 4.7 & 文县-武都 & 1 & 9 & 2 & 0 & 1 \\
\hline 10 & 20000606 & 5.9 & 景泰 & 0 & 11 & 9 & 2 & 10 \\
\hline 11 & 20021214 & 5.9 & 玉门 & 2 & 16 & 5 & 1 & 2 \\
\hline 12 & 20031025 & 6.1 & 民乐-山丹 & 10 & 553 & 26 & 117 & 13 \\
\hline 13 & 20031113 & 5.2 & 㞾县-临潭 & 1 & 133 & 14 & 31 & 16 \\
\hline 14 & 20040907 & 5 & 峔县-卓尼县 & 1 & 37 & 6 & 2 & 2 \\
\hline 15 & 20060621 & 5 & 武都-文县 & 1 & 7 & 3 & 0 & 1 \\
\hline 16 & 20130722 & 6.6 & 㞾县-漳县 & 95 & 4132 & 52 & 864 & 15 \\
\hline
\end{tabular}

灾情应急评估

选取 2013 年 12 月 31 日发布 2014 年 7 月 1 日实施的地震灾情应急评估中规范性 附录中人员伤亡估算方法, 公式如下:

$$
N_{D}=\sum_{j=6}^{I_{\max }} A_{j} \rho R_{j}
$$

式中: $N_{D}$ 为死亡人数; $I_{\max }$ 为极震区 烈度; $A_{j}$ 为第 $j$ 烈度值分布面积; $\rho$ 为人 口密度; $R_{j}$ 为第 $\mathrm{j}$ 烈度值对应的死亡率, 并 给出了死亡率与烈度的统计关系。

\section{5. 适用性评价}

\section{1 死亡人数预测值与实际值比较}

本文基于 4 类评估模型对篮选出的 16
文采用平均相对误差和均方根误差来衡量 估算值与真值之间的偏差, 如表 2 所示。 整体准确性来看, Badal 和刘金龙提出的地 震灾害生命损失评估模型在甘肃省历史震 例验证中效果较好。

表 2 四种模型评估结果的平均相对误差和均方根

\begin{tabular}{|c|c|c|c|c|}
\hline 模型 & 刘吉夫 & Badal & $\begin{array}{c}\text { GB/T30352- } \\
2013\end{array}$ & 刘金 \\
\hline 平均 & & $81 \%$ & $142 \%$ & $96 \%$ \\
\hline 均方根误差 & 1019.46 & 12.58 & 194.18 & 20.70 \\
\hline
\end{tabular}
误差

\section{2 死亡人数预测值与实际值比较}

对上述死亡人数预测值与实际值比较结 果分析, 发现: 
(1) 将震例分三个震级档分别利用均方根 误差来度量模型预测值与实际死亡数值的 相对差异程度, 如表 3 所示。震级 $M s<5.5$ 、 $5.5 \leqslant \mathrm{Ms} \leqslant 6.0$ 级地震 Badal 提出的以人口 密度划分的生命损失评估模型、刘金龙提 出的基于震中烈度的生命损失评估模型以 及国家地震灾情应急评估模型相对较为准 确, 对地震应急响应级别判定具有参考意 义以及对应急决策部署可以起到科技支撑 作用。而刘吉夫提出的以人均 GDP 划分标 准的生命损失评估模型评估结果与实际值 基本不在一个数量级上且整体数值偏大, 该模型在对人均 GDP 的划分标准时可能不 适用甘肃地区, 从而造成评估结果的较大 误差, 总体来说在实际地震死亡人数评估 时基于宏观生命易损性研究方法可能对甘 肃部分地区不适用。

表 3 四种模型在不同震级档中评估结果误差比较

\begin{tabular}{ccccc}
\hline \multirow{2}{*}{ 震级档 } & \multicolumn{4}{c}{ 均方根误差 } \\
\cline { 2 - 5 } & \multirow{3}{*}{ 刘吉夫 } & Badal & GB/T30352- & 刘金 \\
& & & 2013 & 龙 \\
\hline$M s<5.5$ & 53.34 & 6.09 & 11.49 & 5.79 \\
$5.5 \leq M s \leq 6.0$ & 45.44 & 4.44 & 1.61 & 4.39 \\
$M s>6.0$ & 2352.04 & 26.79 & 448.10 & 46.57 \\
\hline
\end{tabular}

震级 Ms $>6.0$ 级的地震评估准确性明显 不如 6 级以下地震评估结果, 部分评估结 果与实际结果的差异超过 1.5 倍, 存在较 大的偶然性。造成这种明显差异可能是由 于随着震级的提高, 影响地震人员死亡的 因素增加或者某一因素得到增强, 因而特 殊的震例需要相应特定适用范围的模型进 行评估, 在实际地震发生后的死亡人数评 估需要在模型评估的基础上综合专家经验 综合判断给出结论。

（2）地震灾害人员伤亡情况在甘肃省分 区现象并不明显, 但因河东地区多为山地, 山高谷深, 地势险峻, 一般地震后发生的 地震灾害 (滑坡、崩塌、泥石流) 较多, 从而导致人员伤亡比河西地区多一些 ${ }^{[18]}$ 。 例如, 2013 年甘肃岷县漳县 6.6 级地震造 成人员死亡 95 人, 其中因地震次生灾害造 成人员死亡 14 人。本文将样本震例分为河 东河西两区, 分别计算 4 种模型评估结果 均方根误差, 检验其精度, 发现河东地区
发生地震模型评估结果准确性与河西地区 相比差异较大, 如图 1 所示。造成这种明 显差异可能由于地震造成人员伤亡的区域 性影响因素增加, 一般地震震级与人员伤 亡成正比, 但个别地震因地震发生地点不 同而有所不同, 主要影响因素可能与人口 密度、地理环境、发震时间、建筑结构等 有关, 因此在和河东地区进行生命损失评 估时需要综合考虑区域特征并结合专家经 验给出评估结论。在地震灾害生命损失评 估模型研究中, 构建针对区域性地震生命 损失特征的典型评估模型尤为重要。

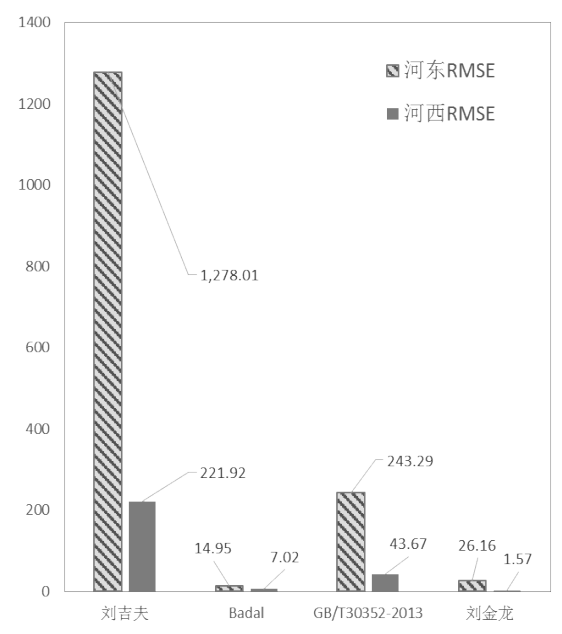

图 1 甘肃河东河西两区 4 种模型评估结果 均方根误差比较

\section{6. 结论与讨论}

\section{1 结论}

破坏性地震发生后, 及时、高效、有序 地进行伤亡人员救助, 减少人员伤亡是震 后抢险救灾的首要任务。投入的应急救灾 力量与人员伤亡数量密切相关, 因此解决 地震死亡人口快速评估并明确结果的可信 度具有重要的研究意义和实用价值。本文 以地震灾害生命损失评估模型适用性对比 为研究内容, 得到以下几点结论:

(1) 从理论方面, 基于地震参数的经 验公式基于统计规律, 对历史数据的依赖 性比较强, 选取的历史数据集合不同对建 
立的计算方法会产生很大影响, 但简单的 参数获取比较适用于震后人员伤亡快速评 估的需要。因此, 在应对震后人员快速评 估的需要时, 经验公式是一个比较理想的 模型。

（2）从实际应用角度, 基于 4 类地震 灾害生命损失评估模型对甘肃地区 16 次地 震的人员死亡情况进行评估, 并将评估结 果与地震实际造成的人员死亡数量比较, 结果表明:

(1)震级 $M_{s}<5.5 、 5.5 \leqslant M_{s} \leqslant 6.0$ 的级地 震 Badal 提出的以人口密度划分的生命损 失评估模型、刘金龙提出的基于震中烈度 的生命损失评估模型以及国家地震灾情应 急评估模型相对较为准确, 对地震应急响 应级别判定具有参考意义以及对应急决策 部署可以起到重要支撑作用。震级 $M s>6.0$ 级地震由于本身的震例资料较少, 导致统 计拟合的样本数偏少, 造成了拟合的离散, 产生误差, 需对评估区域的自然地理、社 会经济、特定风俗等方面存在的明显特征 进行分析得到综合评估结果来支撑应急决 策。

(2)虽然这些模型给出的公式方法基本符 合回归拟合的要求, 研究结果通过统计分 析得到, 但大多模型受限于某一特定的适 用范围或输入条件的限制性, 评估结果也 存在明显的区域性: 甘肃河东地区发生地 震模型评估结果准确性不如河西地区。在 地震灾害生命损失评估模型研究中, 构建 针对区域性地震生命损失特征的典型评估 模型尤为重要。

\section{2 讨论}

从实际应用角度来看, 地震生命损失快 速评估模型是地震应急救援决策部署的重 要组成部分, 评估模型类别很多, 但在地 震行业应急中心等部门实际应用的较少。 破坏性地震一旦发生, 国家台网中心和地 震所在省地震局会在震后十分钟左右向社 会发布地震三要素 (时间、地点和震级), 并将可能造成的地震灾情上报政府, 政府 将依据地震灾害级别迅速启动相应级别的 地震应急救援。如何输入较少预估参数、
减少人为主观因素、提高地震生命损失快 速评估结果的可信度是未来需要迫切解决 的科学问题, 而往往评估模型的建立是在 准确知道重灾区范围、灾区范围、震中烈 度及受灾人口的基础之上, 这对整个地震 应急指挥技术系统的部署提出了更高的要 求。

提高地震灾害生命损失评估模型精度, 更好地服务于各级政府地震灾害应急指挥 决策, 应从以下三方面开展研究: (1)开展 区域基础灾情数据的收集与整理工作, 重 视灾情数据的综合汇集与分级分类管理标 准, 研究建立基础灾情数据收集的标准和 有效的信息资源共享机制, 为构建生命损 失快速评估模型奠定数据基础; (2)地震后 生命易损性模型中涉及生命损失的影响因 素众多, 现实中无法考虑所有影响因素并 统计出人员伤亡与各个因素之间的关系, 从而得出普适性的经验统计公式, 这是非 常困难的。根据实际需求, 在详细分析地 震灾害生命损失时空特征基础上, 有针对 性的建立区域评估模型从而改善或解决评 估模型精度问题, 如建立区域性模型或者 分震级建立评估模型; (3)现有精度较高的 模型参数复杂, 评估数据要求很高, 实际 应用时数据很难收集与更新, 实用性不强。 实际应用的生命易损性模型应具备输入较 少参数、评估所需基础数据收集与更新容 易、模型评估结果科学合理, 建立简单实 用的生命易损性模型需要做进一步的研究。

\section{Acknowledgements}

This study was supported by The Spark Program of Earthquake Technology of CEA (XH18058) and Science and Technology Support Program of Gansu Province(No. 1504FKCA065)

\section{致谢}

本研究得到地震科技星火计划 (XH18058) 和甘肃省科技支撑项目（1504FKCA065）共 同资助。 


\section{参考文献}

[1] 刘百簏, 张俊玲, 吴建华,等. 1920 年 12 月 16 日海原 8.5 级大地震的伤亡人口 再评估. 中国地震, 19(4):386-399, 2003.

[2] ChenJ, Li W, Chen W K, Zhang S P. Assessment of earthquake prevention and disaster reduction capability of countylevel administrative units in Gansu Province. Journal of Risk Analysis and Crisis Response, 7(4): 214-224, 2017.

[3] 李媛媛, 苏国峰, 翁文国, 等. 地震人员伤 亡评估方法研究. 灾害学, 29(2):223-227, 2014.

[4] 尹之潜. 地震灾害损失预测研究. 地震 工程与工程振动, (4):87-96, 1991.

[5] 何玉林, 范灵春, 黎大虎, 等. 四川省地震 灾害预测与评估系统设计及实现功能. 华北地震科学, 23(2):32-36, 2005.

[6] Kircher C A, Whitman R V, Holmes W T. HAZUS Earthquake Loss Estimation Methods. Natural Hazards Review, 7(2):45-59, 2006.

[7] Ploeger S K, Elsabbagh A, Saatcioglu M, et al. Development of the CanRisk earthquake injury model. Natural Hazards, 80(2):1-24, 2016.

[8] Samardjieva E. Estimation of the Expected Number of Casualties Caused by Strong Earthquakes. Bulletin of the Seismological Society of America, 92(6):2310-2322, 2002.

[9] Badal J, Vázquez-Prada M, Álvaro González. Preliminary Quantitative Assessment of Earthquake Casualties and
Damages. Natural Hazards, 34(3):353374, 2005.

[10] Chen Q F, Hongliang M I, Huang J. A Simplified Approach to Earthquake Risk in Mainland China. Pure \& Applied Geophysics, 162(6-7):1255-1269, 2005.

[11] 陈颙,刘杰,陈棋福,等.地震危险性分析 和震害预测.北京:地震出版社, 1999.

[12] 刘吉夫, 陈颗, 史培军, 等. 中国大陆地震 风险分析模型研究( II ): 生命易损性模 型. 北京师范大学学报 (自然科学版), 45(4):404-407, 2009.

[13] 中国地震局震灾应急救援司. 1966-1989 年中国地震灾害损失资料汇编. 地震出 版社, 2015.

[14] 中国地震局, 中国统计局.中国大陆地震 灾害损失评估汇编(1990-1995 年). 北京: 地震出版社. 1996.

[15] 中国地震局震灾应急救援司. 中国大陆 地震灾害损失评估汇编(1996-2000 年). 北京: 地震出版社. 2000.

[16] 中国地震局震灾应急救援司. 中国大陆 地震灾害损失评估汇编(2001-2005 年). 北京: 地震出版社. 2010.

[17] 中国地震局震灾应急救援司. 2006-2010 年中国大陆地震灾害损失评估汇编. 地 震出版社, 2015.

[18] 刘金龙, 林均岐. 基于震中烈度的地震 人员伤亡评估方法研究. 自然灾害学报, (5):113-119, 2012.

[19] 裴惠娟, 周中红, 孙艳萍, 等. 甘肃省地震 灾害时空分布特征研究. 自然灾害学报, (3):67-75, 2015. 